\title{
Piezoelectric Olfactory Receptor Biosensor with Aptamer- assisted Immobilization Technique
}

\author{
Liping $D u^{1}$, He Peng ${ }^{2}$, Chunsheng $W u^{1}$, Luhang Zhao ${ }^{2}$, Ping Wang ${ }^{1 *}$ \\ ${ }^{1}$ Biosensor National Special Laboratory, Key Laboratory for Biomedical Engineering of Ministry of \\ Education, Department of Biomedical Engineering, Zhejiang University, Hangzhou 310027, China \\ ${ }^{2}$ Department of Biochemistry and Genetics, School of Medicine, Zhejiang University, Hangzhou
} 310058, China

\begin{abstract}
This paper presents an olfactory receptor-based piezoelectric biosensor for sensitive odor detection, in which aptamer-assisted olfactory receptor immobilization method is employed for the purpose of performance enhancement. Olfactory receptors (ORs) with a his-tag were employed as the sensitive elements. Anti-his aptamers were immobilized covalently onto the gold surface of piezoelectric quartz crystal microbalance (QCM) electrode to capture his-tagged ORs specifically. By this method, the covalent immobilization as well as the purification of ORs was obtained simultaneously. The results demonstrated that aptamer-assisted OR immobilization technique can improve the performances of the biosensor greatly. Specifically, the detection limit of this OR-based biosensor is as low as 2-3 ppm.
\end{abstract}

Key words: olfactory receptor, piezoelectric biosensor, aptamer, immobilization, odor detection

\section{Background}

Since the concept of "bio-electronic nose" was proposed by Göpel for the first time [1], mimicking nature's olfactory system becomes a new trend to develop novel biosensors for odorant detection. In the biological olfactory system, olfactory receptors (ORs) play a great role in the signal transduction of olfaction and they are suitable to be employed as sensitive elements of biosensors to detect odorants specifically. One of the most crucial issues in the field of olfactory receptor-based biosensors is how to achieve functional immobilization of olfactory receptors with high efficiency on the solid surface of secondary transducers.

Aptamers are single-stranded oligonucleic acid molecules which show a very high affinity to their specific targets and have been widely viewed as promising complementary molecules to antibodies. Compared with antibodies, aptamers have a lot of advantages, such as more resistant, being more easily modified with functional groups or tags, less expensive. [2]

Here we presented an olfactory receptor-based piezoelectric biosensor, in which aptamers were employed to immobilize ORs on the quartz crystal microbalance (QCM), effectively. QCM is a stable and commonly used mass sensitive device. [3] The oscillation frequency changes with the mass loading on the crystal. Therefore, it can be used for monitoring the interaction between odorants and ORs on the crystal.

\section{Materials and Methods}

In this study, an olfactory receptor of C.elegances, ODR-10, was used as a model of ORs. To assist the immobilization of ORs on the QCM sensor, a six-histamine acid his-tag was inserted in the $\mathrm{N}$ terminal of ODR-10 and his-tagged ORs were expressed on the plasma of HEK-293 cells. Then ORs were extracted for the biosensing application.

To immobilize ORs on the QCM sensor effectively, anti-his aptamer was 5'-modified with -SH and a linker - $\left(\mathrm{CH}_{2}\right) 6$ (5'-HS(CH2)6GCTATGGGTGGTCTGGTTGGGATTG GCCCCGGGAGCTGGC-3') and it has a comparable ability to bind the his-tag as antibodies. [4] As shown in Fig. 1(A), anti-his aptamers formed a self assembly monolayer (SAM) on the QCM Au electrode through Au-S bonds to bind the his-tagged olfactory receptors specifically. While other proteins can not bind to the Au electrode due to the lack of his-tag. The immobilization procedure was monitored by electrochemical technique and scanning probe microscopy (SPM). Block molecules (11mercaptoundecanoic, 11-MUA) were used to provide suitable steric spaces for the aptamers and block the free sites. The whole procedure was monitored by electrochemical techniques, 
such as cyclic voltammetry (CV) and electrochemical impedance spectroscopy (EIS).
A commercial QCM sensor detection system is employed and its detection principle is shown in Fig. 1(B).
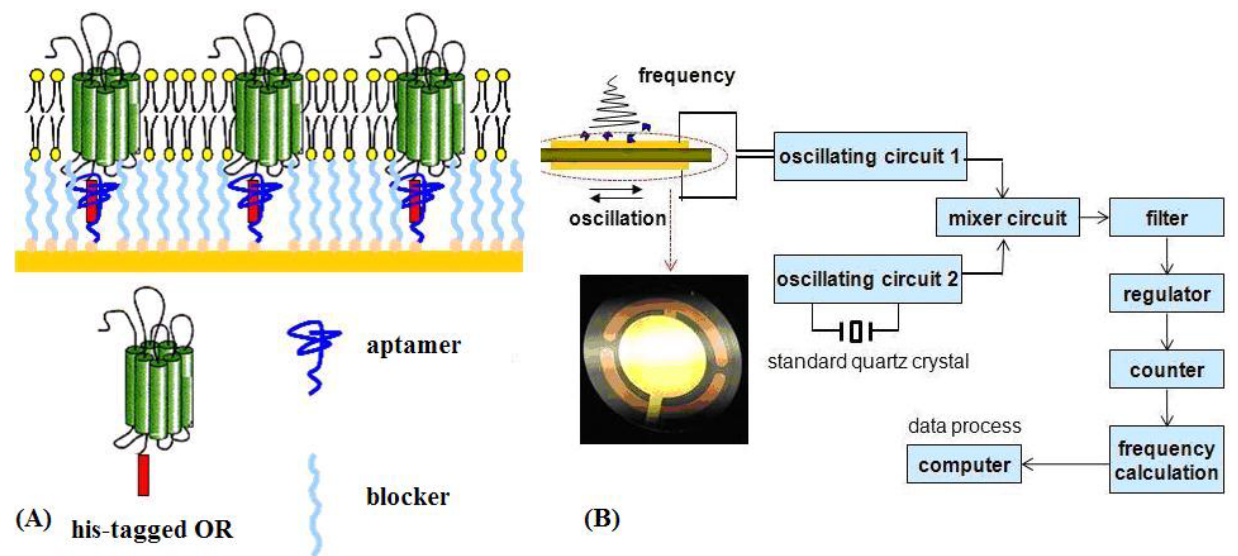

(B)

Fig. 1. (A) A Schematic of aptamer-assisted OR immobilization method. (B) QCM measurement system.

\section{Results and Discussions}

The procedure of ODR-10 immobilization on the surface of QCM gold electrodes was monitored by $C V$ and EIS. As shown in Fig. 2(A), a clean gold electrode gives a reversible cyclic voltammogram (curve a). After the modification of aptamers and block molecules, a mixed SAM was formed on the surface of QCM, accompanying by a considerable decrease in amperometric responses of the electrode and an increase in the peak to peak separation between the cathodic and anodic waves of the redox probe (curve b). This indicates the formation of SAM resulted in an insulating surface and the electron transfer kinetics are perturbed. When ODR-10 tagged with his-tag was captured by the aptamers, a slight current response was observed (curve $\mathrm{c}$ ). This is probably due to the conformation changes of aptamers and structural re-arrangement on the electrode after binding with the olfactory receptor proteins. The current increase indicates that olfactory receptor proteins are captured by aptamers and the stable immobilization is formed on the surface of electrode.

Nyquist plots of impedance spectra of the immobilization procedure are shown in Fig. 2(B), in which significant differences between each step of immobilization were observed and there was the same change trend as CV observed. The impedance data was fitted with a commercial available software Zview/Zplot and the equivalent circuit was shown in the inset of Fig. 2(B).
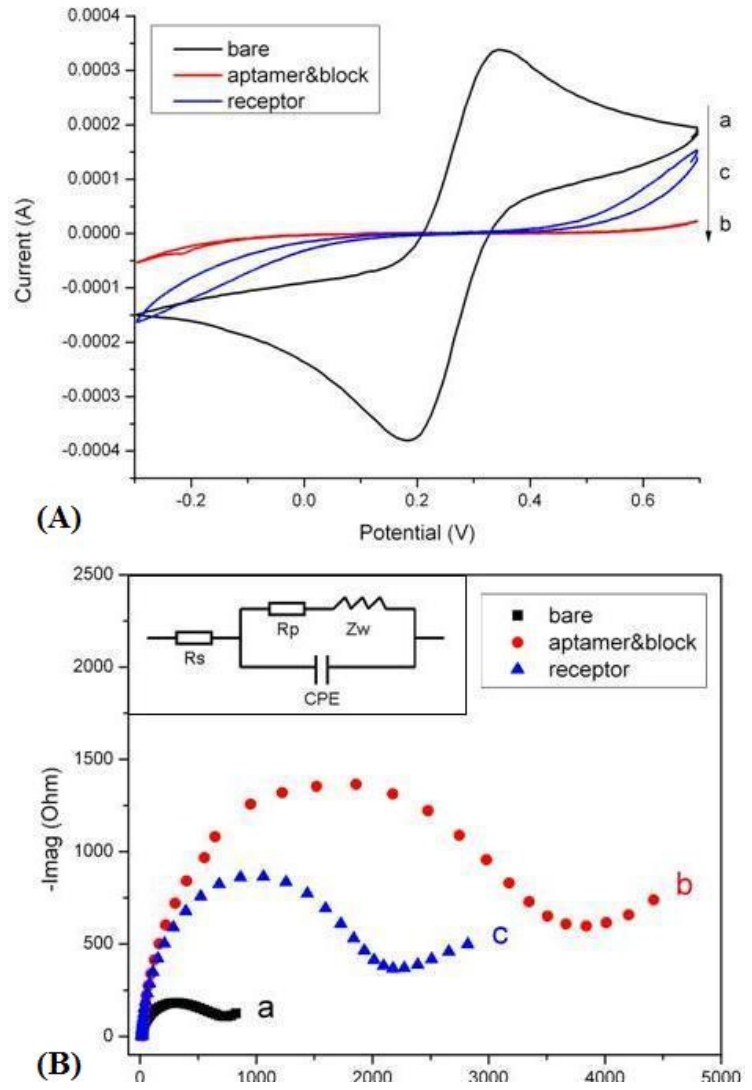

Fig. 2 Electrochemical characteristics of the immobilization procedure. (A) CV measurements. a: bare gold electrode; $b$ : aptamer modification and 11MUA blockage; c: olfactory receptor immobilization.

(B) Nyquist plots of impedance spectra in the frequency range from $1 \mathrm{~Hz}$ to $100 \mathrm{kHz}$. The inset of Fig. 2(B) shows the equivalent circuit model.

SPM was used to characterize the surface morphology of ODR-10 immobilization. Gold electrodes functionalized with ODR-10 captured by aptamers were used as samples. As presented in Fig. 3, evenly distributed grainy features can be observed. The sizes of grainy 
features range from $30 \mathrm{~nm}$ to $100 \mathrm{~nm}$ and the height is around $6 \mathrm{~nm}$. These sizes are consistent to theoretical values. In addition, the thickness of the receptor-containing layer is approximately equivalent to the thickness of the cell membrane, indicating that membrane vesicles open upon immobilization. It was also found that membrane fractions were distributed in some areas of the electrode, which indicates that the olfactory receptor is immobilized in membrane fraction. This could provide hydrophobic environment for the immobilized olfactory receptors to facilitate the maintaining of their natural structures and functions.

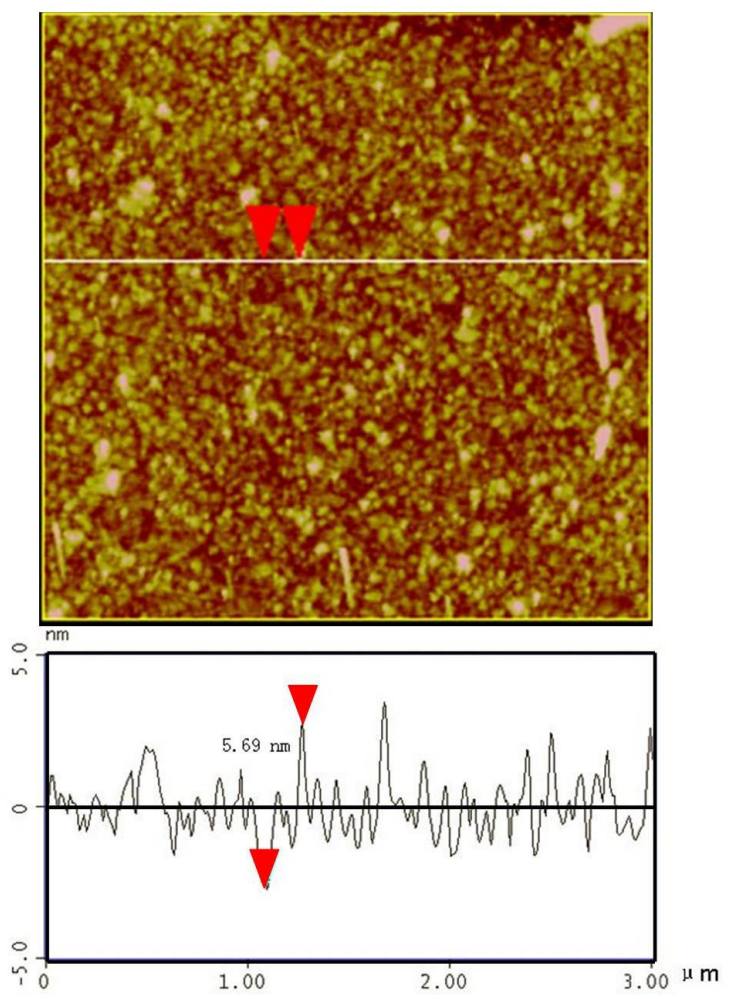

Fig. 3 SPM image $(3 \mu m \times 3 \mu m)$ of QCM surface after the immobilization of OR.

We applied this aptamer-based immobilization method to the development of olfactory receptor-based QCM biosensor for odorant detection. ODR-10 was functional immobilized on the gold electrode of QCM by aptamerbased method. The responses of this QCM biosensor to various odorants were monitored by recording the resonance frequency shifts of QCM. Fig. 4 shows the results of this QCM biosensor responding to various odorants. Fresh air was used to detect the influence of the air flow and served as negative control. The frequency shifts were normalized to that of diacetyl. The results show that the responses of this QCM biosensor to diacetyl are significantly higher than that of other odorants tested. It is indicated that this QCM biosensor can specifically responds to the natural ligand of ODR-10, diacetyl. It is demonstrated that the aptamer-based immobilization method can be successfully applied in the development of olfactory receptor-based QCM biosensors. In future, based on this idea, various olfactory receptors can be tagged by different tags and captured by the corresponding aptamers on the electrode surface. By this method, molecular sensor arrays of olfactory receptor can be developed for high sensitive and highthroughput detection of specific odorants.

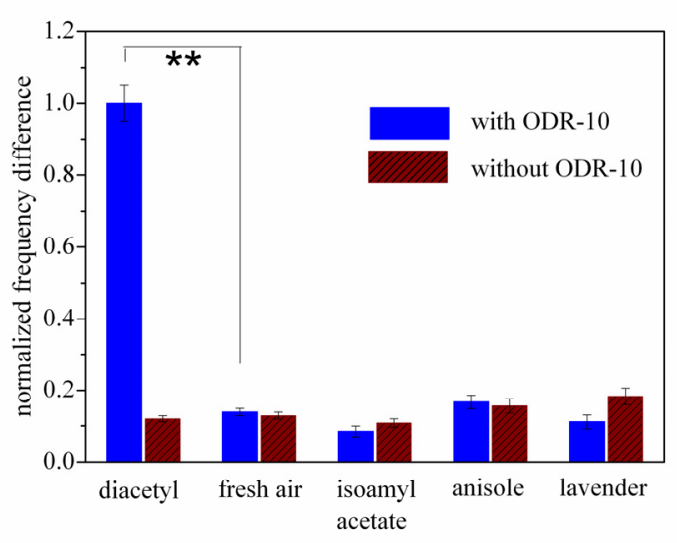

Fig. 4 Responses of OR-based QCM biosensor to various odorants. All the data are represented by means $\pm S D$ (standard deviation). ${ }^{* *} p<0.05, n=4$, student's t-test.

The sensitivity of this biosensor was determined by measuring various concentrations of diacetyl. The results demonstrate that the detection limit of this OR-based biosensor is as low as 2-3 ppm. The biosensor also exhibits good reproducibility and repeatability to diacetyl. After seven days storage in room temperature, the responses of biosensor decreased only $10 \%$.

\section{Conclusions}

In this study, a piezoelectric olfactory receptor biosensor was presented, in which aptamers were employed to assist the functional and high efficient immobilization of olfactory receptors. The results demonstrated that this apatmerbased method can greatly simplify the development of olfactory receptor-based biosensors and improve the immobilization efficiency. The olfactory receptor-based QCM biosensor also has good performance for odorant detection. It is suggested that this aptamer-assiting protein immobilization method can improve the performance of biosensors greatly, and has great potential to be applied in many biosensing applications, especially for the membrane receptor-based biosensors due to the high efficient immobilization and purification 
function of aptamers. In addition, olfactory receptor-based QCM biosensors can be used in many fields, such as food safety, environment monitoring, and poison gas detection.

\section{Acknowledgements}

This work was supported by the grants from the National Natural Science Foundation of China (Grant Nos. 60725102, 31000448), the China Postdoctoral Science Foundation (Grant No. 20100471737) and the Key Project of Zhejiang Province (Grant No. 2010C14006).

\section{References}

[1] W.Göpel, Ch.Ziegler, H.Breer, D.Schild, R.Apfelbach, J.Joerges, R.Malaka, Bioelectronic noses: a status report - Part 1,Biosensors and Bioelectronics 13, 479-493(1998); doi: 10.1016/S0956-5663(97)00092-4

[2] T. Mairal, V.C. Özalp, P.L. Sanchez, M. Mir, I. Katakis, C.K.O' Sullivan, Aptamers:molecular tools for analytical applications. Analytical and Bioanalitical chemistry 390(4), 989-1007(2008); doi: 10.1007/s00216-007-1346-4

[3] S. Sankaran, S. Panigrahi, S. Mallik. Olfactory receptor based piezoelectric biosensors for detection of alcohols related to food safety applications. Sensors and Actuators, B-Chemical 155(1): $8-18$ (2011); doi:10.1016/j.snb.2010.08.003

[4] J.G. Walter, Ö. Kökponar, K. Friehs, F. Stahl, T. Scheper, Systematic investigation of optimal aptamer immobilization for protein-microarray applications, Analytical Chemistry 80(19), 73727378(2008); doi: 10.1021/Ac801081v 